\title{
MINS, a Novel Naphthalimide-Polyamine Conjugate, Induced Apoptosis Depending on p53 Status in Human Colon Cancer Cells
}

\author{
Ming Li ${ }^{1}$, Tao Yang1, Fujun Dai' ${ }^{2}$, Qian Li ${ }^{2}$, Yahong Zhang², Songqiang Xie ${ }^{1 *}$, \\ Chaojie Wang ${ }^{*}$ \\ ${ }^{1}$ Institute of Chemical Biology, Pharmaceutical College of Henan University, Kaifeng, China \\ ${ }^{2}$ The Key Laboratory of Natural Medicine and Immuno-Engineering, Henan University, Kaifeng, China \\ Email: xiesq@henu.edu.cn, ${ }^{*}$ wcjsxq@henu.edu.cn
}

Received 15 July 2014; revised 20 August 2014; accepted 9 September 2014

Copyright (C) 2014 by authors and Scientific Research Publishing Inc.

This work is licensed under the Creative Commons Attribution International License (CC BY). http://creativecommons.org/licenses/by/4.0/

(c) (i) Open Access

\begin{abstract}
Natural polyamine is an ideal antitumor drug carrier because of the great requirement difference between cancer cells and normal cells. Previous data demonstrated that many cytotoxic drugs conjugated with natural or synthetic polyamines have potent antitumor effects. Up to now, the antitumor mechanism of conjugates of naphthalimides with polyamine remains poorly understood in human colon cancer cells. The aim of this study is to evaluate the effect of MINS (a novel naphthalimide-polyamine conjugate) and mechanism of MINS in human colon cancer cells. Mitochondrial toxicity, which was generated by ROS from mitochondria electron transport chain, might be a major factor in MINS-inducted apoptosis. Our data also demonstrated that MINS-mediated cell apoptosis depend on p53 status, for MINS induced Caco-2 cells (p53 null) only necrosis but not apoptosis. Furthermore, the apoptotic effect of MINS is stronger than that of Amonafide, the parent drug of MINS. Our data suggested that the MINS-mediated cell apoptosis depend on p53 in human colon cancer cells.
\end{abstract}

\section{Keywords}

Naphthalimide-Polyamine Conjugates, Apoptosis, p53, Mitochondrial Transmembrane Potential, Oxidative Stress

\section{Introduction}

Colon cancer is one of the most common malignant tumors in the world with approximately one million new

${ }^{*}$ Corresponding authors.

How to cite this paper: Li, M., Yang, T., Dai, F.J., Li, Q., Zhang, Y.H., Xie, S.Q. and Wang, C.J. (2014) MINS, a Novel Naphthalimide-Polyamine Conjugate, Induced Apoptosis Depending on p53 Status in Human Colon Cancer Cells. Open Journal of Apoptosis, 3, 59-69. http://dx.doi.org/10.4236/ojapo.2014.34007 
cases diagnosed every year [1]. The structure of DNA in normal cells and tumor cells has many differences, so DNA become targets for anticancer drugs and DNA intercalating agents play an important role in antineoplastic chemotherapy [2]. Naphthalimide compounds as DNA intercalating agents exert potent antitumor effects in a variety of murine and human tumor cell lines [3].

The natural polyamines, putrescine (Put), spermidine (Spd) and spermine (Spm), play an essential role in cellular growth and differentiation [4] [5]. The raised polyamine levels are closely linked with tumorigenesis. The polyamines induce the transformation from the normal cells to the malignant tumors by affecting cell proliferation and differentiation, and they also promote the development of tumors by promoting the synthesis of nucleic acids, some biological macromolecules and the formation of tumor blood vessels [6].

Some polyamine analogues and conjugates exerted potent antitumor effects [7]. In recent years, we are committed to the researches of the synthesis and antitumor molecular mechanism of naphthalimide-polyamine conjugate. Most of the naphthalimide-polyamine conjugates exert potent antitumor effect via inducing apoptosis and/or autophagy [8] [9]. Up to now, the antitumor mechanism of conjugates of naphthalimides with polyamine remains poorly understood in human colon cancer cells. To explore the antitumor mechanism of conjugates of naphthalimides with polyamine in human colon cancer cells, we synthesized MINS, a novel naphthalimide-polyamine conjugate, in our laboratory and evaluated the apoptotic molecular mechanism in human colon cancer cells. Here, we first reported that apoptosis might be the terminal fate of human colon cancer cells treated with MINS. Mitochondrial toxicity, generated by ROS from mitochondria electron transport chain, might be a major factor in MINS-inducted apoptosis. Our data demonstrated that the MINS-mediated cell apoptosis depend on p53 condition, and the apoptotic effect of MINS is stronger than that of Amonafide which is the parent drug of MINS. Thus, MINS merits further investigation as a potential chemotherapeutic agent against colon cancers.

\section{Materials and Methods}

\subsection{Materials}

DMEM and fetal calf serum (FCS) were purchased from Gibco (Grand Island, NY, USA). McCoy's 5A, DMSO, Hoechst 33342, JC-1, rhodamine123 (Rh123), propidium iodide (PI) and 3-(4,5-dimethylthiazol)-2,5-diphenyltetrazolium bromide (MTT), pifithrin- $\alpha$, reactive oxygen species (ROS) assay kit, Rotenone, Apocynin and n-acetyl-l-cysteine (NAC) were purchased from Sigma (St. Louis, MO, USA). Annexin V-FITC and Annexin V-PE apoptosis detection kit were purchased from Cell signaling Technology (Beverly, MA, USA). Z-DEVDFMK, Z-IETD-FMK, Z-LEHD-FMK, and Primary antibodies: caspase-3, caspase-8, caspase-9, $\beta$-Actin, HSP60, Bcl-2, Bax, cytochrome $c$, p53, p21, as well as horseradish peroxidase-conjugated anti-mouse or anti-rabbit antibodies were purchased from Santa Cruz Biotechnologies (Santa Cruz, CA, USA). All other chemicals used in the experiments were commercial products of reagent grade. MINS (purity: 99.2\%) was synthesized in our laboratory and the structure formula was shown in Figure 1(A).

\subsection{Amplification and Extraction of pEGFP-N1-p53 Plasmid and Cell Transfection}

Recombinant pEGFP-N1-p53 plasmids were purchased from Promega Beijing Biotech Co., Ltd (Beijing, China) and grown in Escherichia coli. After abundant amplification, the plasmid was purified using a plasmid extraction kit (Axygen Biosciences, CA, USA) and was suspended in $2.5 \mathrm{mM}$ Tris-HCl (pH 8.5) at a concentration of $1.0 \mu \mathrm{g} / \mu \mathrm{L}$. For transfection, Caco-2 cells were seeded in 6 -well plates and incubated at $37^{\circ} \mathrm{C}$ in a humidified incubator containing $5 \% \mathrm{CO}_{2}$. The next day, when these cells were about $80 \%$ confluence, cells were transfected with Transfection Reagent according to the manufacturer's instructions. Briefly, DNA (1.0 $\mu \mathrm{g})$ and $3.0 \mu \mathrm{lof}$ GenJet Ver. II reagents (SignaGen, MD, USA) were diluted with serum free DMEM, respectively, and then the diluted GenJet Ver.II reagents were added to the diluted DNA and the formed transfection complex was kept at RT for 15 minutes. These transfected cells were selected with $0.1 \mathrm{mg} / \mathrm{mL} \mathrm{G418}$ for 8 weeks, and then the stable plasmid-transfected Caco-2-p53 cells were confirmed by RT-PCR and Western Blot (Supplementary Figure 2(A) and Supplementary Figure 2(B)).

\subsection{Cell Culture and Treatment}

HT-29 cell line, HCT-116 cell line and Caco-2 (p53 null) cell line were purchased from Shanghai Institute for Biological Science, Chinese Academy of Sciences (Shanghai, China). Caco-2 cells, Caco-2-p53 cells with stably 


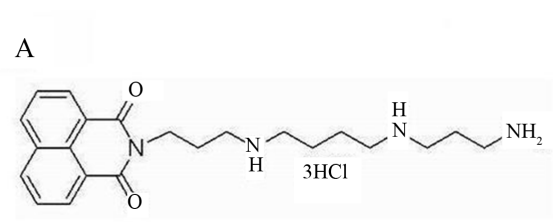

2-\{3-[4-(3-Aminopropylamino)butylamino]propyl $\} 1 \mathrm{H}-$ benz -[de]isoquinoline-1,3(2H)-dione-trihydro-chloride MINS
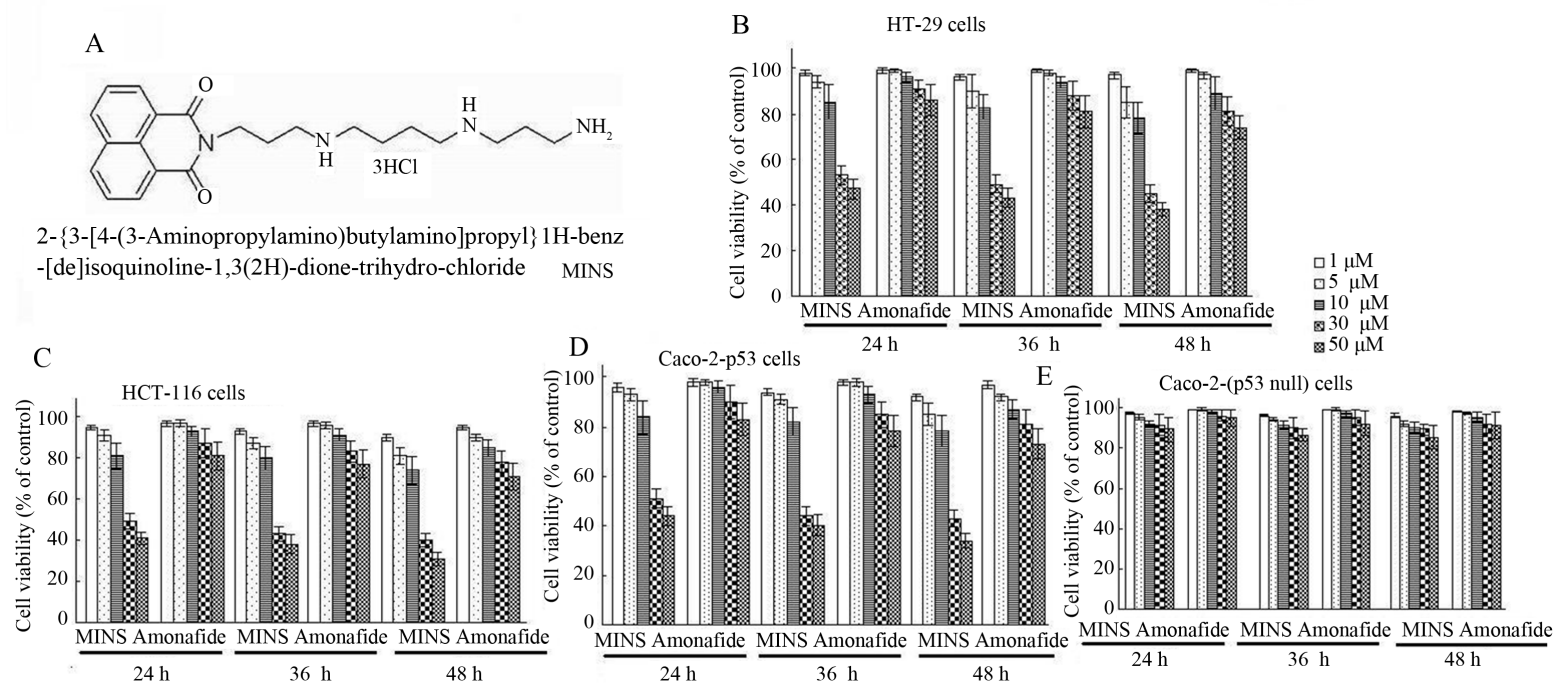

Figure 1. The chemical structure of MINS (A) and anti-proliferation effects of MINS and Amonafide on HT-29 cells (B), HCT-116 cells (C), Caco-2-p53 cells (D) and Caco-2 (p53 null) cells (E) by MTT assay (means \pm SD, $n=3$ ).

transfected p53, and HCT-116 cells were cultured in DMEM supplemented with 10\% FCS (McCoy's 5A for HT-29 cells). $1 \mathrm{mM}$ aminoguanidine was added as an inhibitor of amine oxidase derived from FCS and had no effect on the various parameters of the cells measured in this study [8].

\subsection{Antiproliferative Assay}

The antiproliferative effect of MINS was evaluated in HT-29 cells, HCT-116 cells, Caco-2 (p53 null) cells and Caco-2-p53 cells by the conversion of MTT to a purple formazan precipitate [8]. Briefly, cells were seeded into 96-well plates at $5 \times 10^{3}$ cells/well. After $24 \mathrm{~h}$, various concentrations of MINS were subsequently added and incubated for $48 \mathrm{~h}$. The inhibitive rate was calculated from plotted results using untreated cells as $100 \%$.

\subsection{Cell Apoptosis Evaluation}

Cell apoptosis was evaluated in HT-29 cells, HCT-116 cells, Caco-2 (p53 null) cells and Caco-2-p53 cells by high content screening (HCS) (Thermo Scientific Cellomics ArrayScan Vti, Cellomics, Inc., Pittsburgh, PA) analysis. Firstly, cells were seeded into 96-well plates at $5 \times 10^{4}$ cells/well. After $24 \mathrm{~h}, \mathrm{HT}-29$ cells were treated with MINS for $24 \mathrm{~h}, 36 \mathrm{~h}$ or $48 \mathrm{~h}$. Then the cells were centrifugated with horizontal centrifuge for $5 \mathrm{~min}$, stained with propidium iodide $(50 \mu \mathrm{g} / \mathrm{mL})$ in the dark for $60 \mathrm{~min}$ at $4^{\circ} \mathrm{C}$ and washed 3 times with phosphate-buffered saline (PBS). Secondly, cells were stained with Annexin V-FITC in the dark for $15 \mathrm{~min}$ at room temperature, then washed 2 times with Annexin V-FITC binding solution. Briefly, Annexin V-FITC stock solution $(1 \mu \mathrm{L})$ was added to Annexin V-FITC binding solution $(20 \mu \mathrm{L})$. Thirdly, cells were stained with Hoechst $33342(10 \mu \mathrm{g} / \mathrm{mL})$ in the dark for $15 \mathrm{~min}$ at room temperature, and washed 3 times with PBS. Finally, $50 \mu \mathrm{L}$ PBS was added in every well. The analysis was done using Thermo Scientific Cellomics ArrayScan Vti (MA, USA). Because Caco-2-p53 cells in this study had been transfected green flourescence, so we used AnnexinV-PE with red fluorescene and PI, respectively. The experimental procedures referred to the above three staining methods. Annexin V-positive cells with nuclear shrinkage, blebbing, and apoptotic bodies were counted as apoptotic. The percentage of apoptotic cells was calculated after observing a total of 300 cells. For the p53 inhibitor analysis, the cells were pretreated with PFT- $\alpha(10 \mu \mathrm{M})$ for $2 \mathrm{~h}$ respectively, and then exposed with MINS.

\subsection{Caspase Activity Assay}

The activity of caspases was detected as our previous description [10]. Briefly, the cells, after treatment with MINS for $12 \mathrm{~h}$, were harvested and lysed and then the cytosolic fraction was obtained by centrifugation at 12 $000 \times g$ at $4^{\circ} \mathrm{C}$ for $20 \mathrm{~min}$. DEVDase, IETDase, and LEHDase activities were evaluated by measuring proteo- 
lytic cleavage of chromogenic substrates Ac-DEVD-pNA, Ac-IETD-pNA, and Ac-LEHDpNA, which were used as the substrates for caspase-3, caspase-8, and caspase-9-like proteases, respectively. Briefly, the cell lysate (50 mg of protein) was added into the buffer containing $150 \mathrm{~mol} / \mathrm{L}$ Ac-DEVD-pNA, Ac-IETD-pNA, and AcLEHD-pNA to a final volume of $150 \mu \mathrm{L}$. The reaction mixture was incubated at $37^{\circ} \mathrm{C}$ for $1 \mathrm{~h}$. The absorbance of enzymatically-released pNA was measured at $405 \mathrm{~nm}$ on a microplate reader every $20 \mathrm{~min}$. For the caspase inhibitor analysis, the cells were pretreated with Z-DEVD-FMK $(20 \mu \mathrm{M})$, Z-IEFD-FMK $(20 \mu \mathrm{M})$ or Z-LEHDFMK $(20 \mu \mathrm{M})$ for $2 \mathrm{~h}$ respectively, and then exposed with MINS for $12 \mathrm{~h}$.

\subsection{Mitochondrial Membrane Potential Assay}

Mitochondrial membrane potential (MMP) was assessed by the retention of Rh123 (JC-1 for Caco-2-p53 cells), a membrane-permeable fluorescent cationic dye. The uptake of Rh123 by mitochondria is proportional to the MMP. Briefly, cells harvested as above described were incubated with $20 \mu \mathrm{M}$ Rh123 in the dark for 30 min at room temperature. After washing 3 times with PBS, the cells were stained with Hoechst 33342 and then analyzed by HCS [8].

\subsection{Reactive Oxygen Species Assay}

Reactive oxygen specise (ROS) was assessed by ROS assay kit which detect ROS with fluorescent probe DCFH-DA (dihydroethidium for Caco-2-p53 cells). The cells harvested as above described were stained with 10 $\mu \mathrm{M}$ DCFH-DA in the dark for $15 \mathrm{~min}$ at room temperature. After washing 3 times with PBS, the cells were stained with Hoechst 33342 and then analyzed by HCS. For the ROS inhibitor analysis, the cells were pretreated with Rotenone $(25 \mathrm{nM})$, Apocynin $(100 \mu \mathrm{M})$ or NAC $(500 \mu \mathrm{M})$ for $1 \mathrm{~h}$ respectively, and then exposed with MINS for $24 \mathrm{~h}[8]$.

\subsection{Western Blot}

HT-29 cells, HCT-116 cells and Caco-2-p53 cells, treated with MINS for a specific time period, were harvested and washed with PBS. Total cellular protein was isolated using the protein extraction buffer (containing 150 $\mathrm{mM} \mathrm{NaCl}, 10 \mathrm{mM}$ Tris (pH 7.2), 5 mM EDTA, 0.1\% Triton X-100, 5\% glycerol and 2\% SDS). Protein concentrations were determined using the protein assay kit. Cytosolic and mitochondrial fractions were separated by Kit (C3601, Beyotime, Nantong, China) as instruction. Equal amounts of proteins (50 $\mu \mathrm{g} / \mathrm{lane})$ were separated using $8 \%$ or $12 \%$ SDS-PAGE and transferred to PVDF membranes. The membranes were incubated with primary antibodies. After washed with PBS, the membranes were incubated with peroxidase-conjugated goat antimouse or antirabbit secondary antibody, followed by enhanced chemiluminescence staining through the enhanced chemiluminescence system. $\beta$-Actin (43 kDa) and HSP60 (60 kDa) were used to normalize for protein loading [8].

\subsection{Data Analysis}

All data were presented as mean \pm SD and analyzed using Students $t$ test or analysis of variance (ANOVA) followed by $q$ test.

\section{Results}

\subsection{MINS Inhibited the Growth of Human Colon Cancer Cells}

MINS inhibited the growth in a dose- and time-dependent manner in HT-29 cells, Caco-2-p53 cells and HCT116 cells (Figure 1). Amonafide, from which the MINS is derived, had inferior growth-inhibited effects in all three cell lines comparing with MINS. However, MINS had no obvious inhibition on the growth of p53-null cells (Caco-2) at same doses and times, even at $50 \mu \mathrm{M}$ for $48 \mathrm{~h}$ treatment.

\subsection{Apoptosis Induced by MINS in Three Human Colon Cancer Cells}

Compared with control cells, the percentage of annexin V-positive cells was significantly increased after treatment with MINS in HT-29 cells, Caco-2-p53 cells and HCT-116 cells. Furthermore, the apoptotic rate was also 
increased in a time- and dose-dependence manner (Figure 2), suggesting that MINS could induce apoptosis in three human colon cancer cells. To elucidate the molecular mechanism in MINS-mediated cell apoptosis, we first detected the activity of caspase-3, caspase-8 and caspase-9. After treatment with MINS, caspase-3, caspase8 and caspase-9 were activated in HT-29 cells and Caco-2-p53 cells, however, only caspase-3 and caspase-9 were activated in HCT-116 cells (Figure 3). Moreover, Z-DEVD-FMK, Z-IEFD-FMK or Z-LEHD-FMK could inhibit the activation of caspase-3, caspase-8, caspase-9 and cells apoptosis, respectively (Supplementary Figure 1(A) and Supplementary Figure 1(B)), suggesting MINS-mediated cells apoptosis via death receptor pathway and mitochondrial apoptotic pathway in HT-29 cells and Caco-2-p53 cells, and only mitochondrial apoptotic pathway in HCT-116 cells, and the apoptosis was caspase dependent. The MMP of HT-29 cells, Caco-2-p53 cells and HCT-116 cells were decreased significantly after treatment with MINS (Figure 4), and accordingly facilitated cytochrome $c$ release from mitochondria to cytoplasm, respectively (Figure 5). Furthermore, the protein expression of Bax, p53 and p21 was up-regulated and Bcl-2 was down-regulated after treatment with MINS (Figure 5). MINS induced Caco-2 (p53 null) cells necrosis significantly compared with control, while no obvious apoptosis was observed after treatment with potent antiproliferative concentrations (100 $\mu \mathrm{M}$ and $200 \mu \mathrm{M}$ ) (Supplementary Figure 2(C) and Supplementary Figure 2(D)), suggesting that the presence of p53 could influence the MINS-mediated cell death profile.

\subsection{ROS and p53 Are Necessary for MINS-Induced Cell Apoptosis}

Compared with control cells, ROS generation was significantly increased after treatment with MINS in HT-29 cells, Caco-2-p53 cells and HCT-116 cells (Figure 6). NAC, an inhibitor of ROS, not only inhibited MINSmediated ROS generation but also attenuated MINS-induced growth inhibition, cell apoptosis, caspase-3 activation, p53, p21 and Bax up-regulation, and Bcl-2 down-regulation (Figure 7). To further confirm the resource of MINS-mediated ROS, Rotenone and Apocynin, an inhibitor for mitochondria ROS and NADPH oxidase respectively, were respectively pre-incubated before MINS treatment. Rotenone significantly decreased MINS-mediated ROS generation, however, Apocynin only partly attenuated this effect, suggesting that mitochondria was the predominant resource in MINS-mediated ROS generation (Supplementary Figure 1(C)).

To confirm the significance of p53, PTF-a, a specific p53 inhibitor, was used to detect the change of MINSinduced cell apoptosis. After pre-treatment with PTF- $\alpha$, MINS-induced growth inhibition, cell apoptosis, caspase-3 activation, p53, p21 and Bax up-regulation and Bcl-2 down-regulation were obviously attenuated in HT29 cells, Caco-2-p53 cells and HCT-116 cells (Figure 7). Interestingly, co-preincubation of NAC and PTF-a almost completely reversed MINS-mediated cell apoptosis (Supplementary Figure 1(B)). Furthermore, MINS

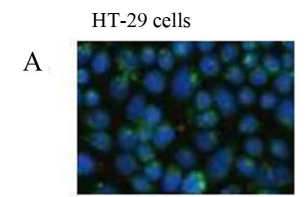

Control $(0 \mu \mathrm{M})$

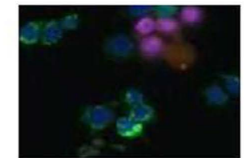

Amonafide $(60 \mu \mathrm{M})$

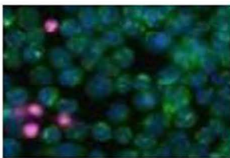

MINS $(25 \mu \mathrm{M})$

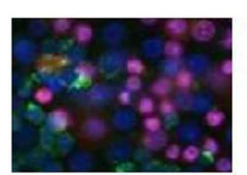

MINS $(35 \mu \mathrm{M})$

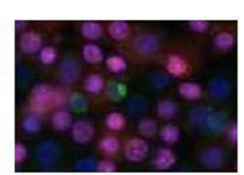

MINS $(45 \mu \mathrm{M})$
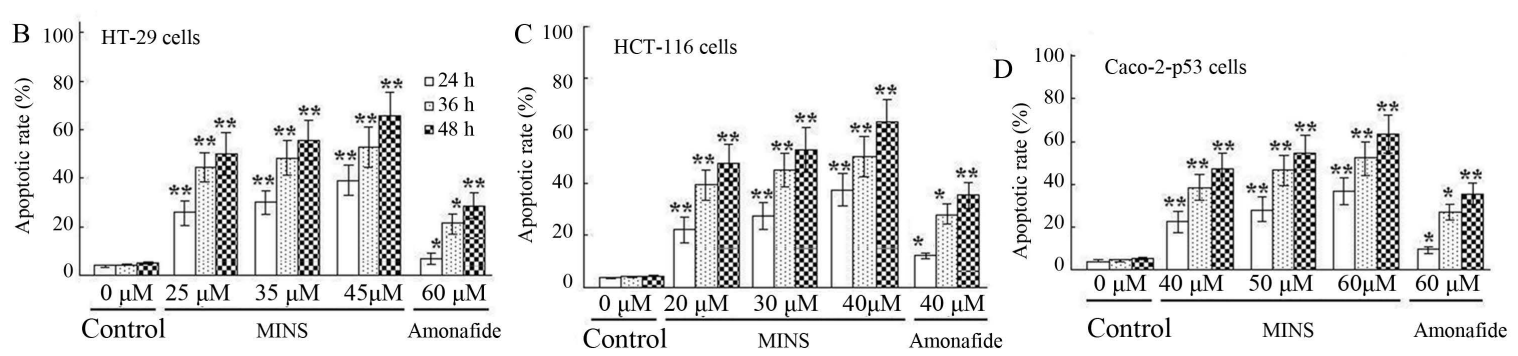

Figure 2. MINS induced HT-29 cells, HCT-116 cells and Caco-2-p53 cells apoptosis. (A) The representative photograph of HT-29 cells with Annexin V-FITC /PI/Hoechst 33342 three staining by HCS (10×). The cellular nucleus were stained by Hoechst 33342 (blue fluorescence), the cellular nucleus of lately apoptotic and necrotic cells were stained by PI (red fluorescence) and the cellular membranes of early apoptotic cell were stained by Annexin V-FITC (green fluorescence). The apoptotic rate change of HT-29 cells (B), HCT-116 cells (C) and Caco-2-p53 cells (D) after treatment with various concentrations of MINS and Amonafide for $24 \mathrm{~h}, 36 \mathrm{~h}$ or $48 \mathrm{~h}$, means $\pm \mathrm{SD}, \mathrm{n}=3$, Note: ${ }^{*} \mathrm{p}<0.05,{ }^{* *} \mathrm{p}<0.01$ vs control. 

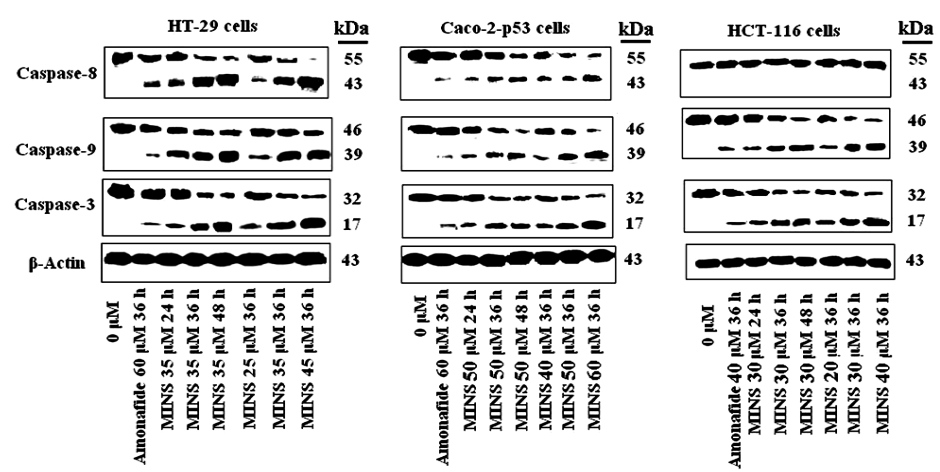

Figure 3. The representative photograph of Caspase-3, -8, -9 expression after treatment with MINS and Amonafide at the indicated doses and times by Western Blot in HT-29 cells, HCT-116 cells and Caco-2-p53 cells. Equal amounts of cellular protein were separated using $8 \%$ or $12 \%$ SDS-PAGE and transferred to PVDF membranes. $\beta$-Actin protein was blotted as a control. The independence experiment was replicated three times.

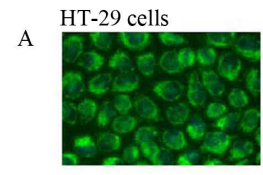

Control $(0 \mu \mathrm{M})$

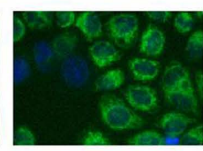

Amonafide $(60 \mu \mathrm{M})$

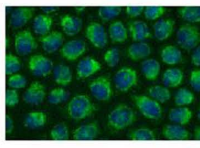

MINS $(25 \mu \mathrm{M})$

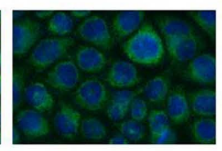

MINS $(35 \mu \mathrm{M})$

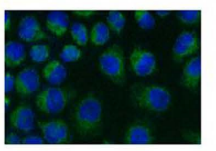

MINS $(45 \mu \mathrm{M})$
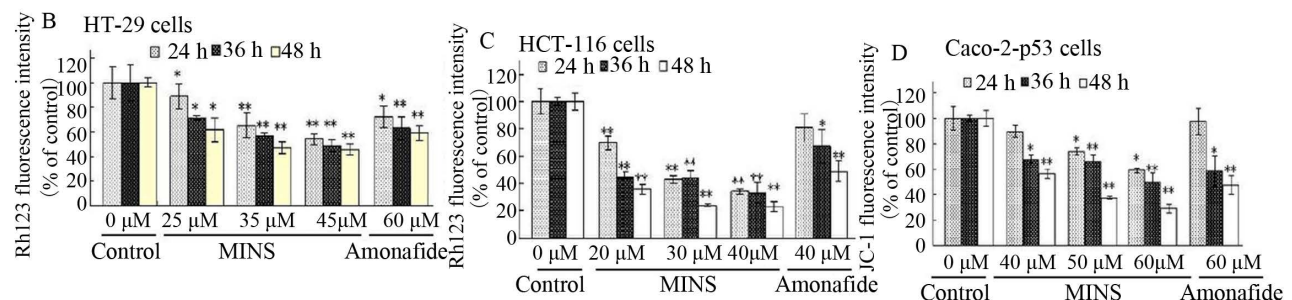

Figure 4. The effect of MINS about Mitochondrial transmembrane potential. (A) The representative photograph of HT-29 cells with Rh 123/Hoechst 33342 double staining by HCS $(10 \times$ ). The cellular nucleus were stained by Hoechst 33342 (blue fluorescence), and the mitochondria were stained by Rh 123 (green fluorescence). The Rh 123 fluorescence intensity (\% of control) changes of HT-29 (B), HCT-116 (C) and Caco-2-p53 cells (D) after treatment with various concentrations of MINS and Amonafide for 24 h, 36 h or 48 h, means \pm SD, $n=3$, Note: ${ }^{*} p<0.05,{ }^{* *} p<0.01$ vs control.
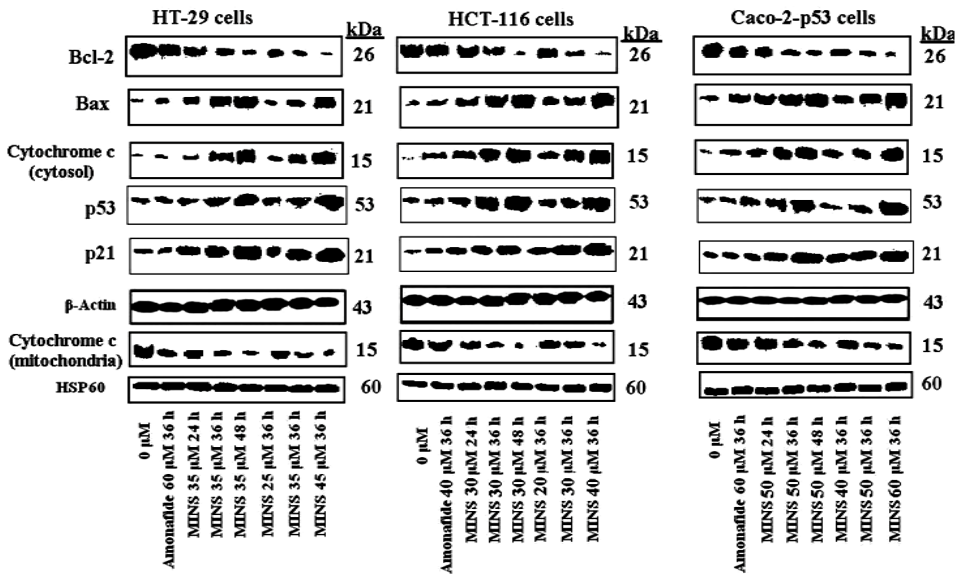

Figure 5. The representative photograph of Bcl-2, Bax, p53, p21 and Cytochrome c expression after treatment with MINS and Amonafide at the indicated doses and times by Western Blot in HT-29 cells, HCT-116 cells and Caco-2-p53 cells. Equal amounts of cellular protein were separated using $8 \%$ or $12 \%$ SDS-PAGE and transferred to PVDF membranes. $\beta$-Actin and GADPH protein were blotted as a control. The independence experiment was replicated three times. 
HT-29 cells

A 2009

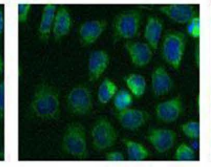

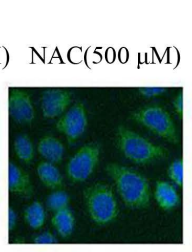
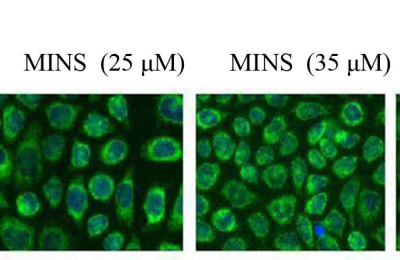

MINS $(35 \mu \mathrm{M})$

$\stackrel{+}{\mathrm{NAC}}(500 \mu \mathrm{M})$

B

HT-29 cells

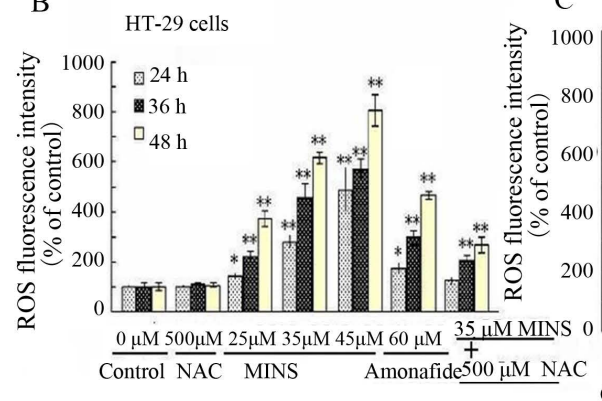

C HCT-116 cells
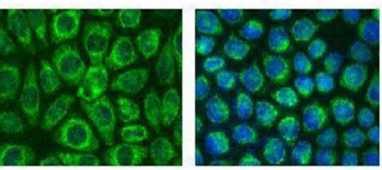

Control NAC MINS Amonafide $500 \overline{\mu M}$ NA

Control NAC $\frac{20 \mu \mathrm{M} 30}{\mathrm{MINS}}$

Figure 6. The effect of MINS on ROS generation. (A) The representative photograph of HT-29 cells with DCFH-DA/ Hoechst 33342 double staining by HCS $(10 \times)$. The cellular nucleus were stained by Hoechst 33342 (blue fluorescence), and the ROS were stained by DCFH-DA (green fluorescence). The DCFH-DA fluorescence intensity (\% of control) changes of HT-29 (B), HCT-116 (C) and Caco-2-p53 cells (D) after treatment with various concentrations of MINS and Amonafide for $24 \mathrm{~h}, 36 \mathrm{~h}$ or $48 \mathrm{~h}$, means $\pm \mathrm{SD}, \mathrm{n}=3$, Note: ${ }^{*} \mathrm{p}<0.05,{ }^{* *} \mathrm{p}<0.01$ vs control.
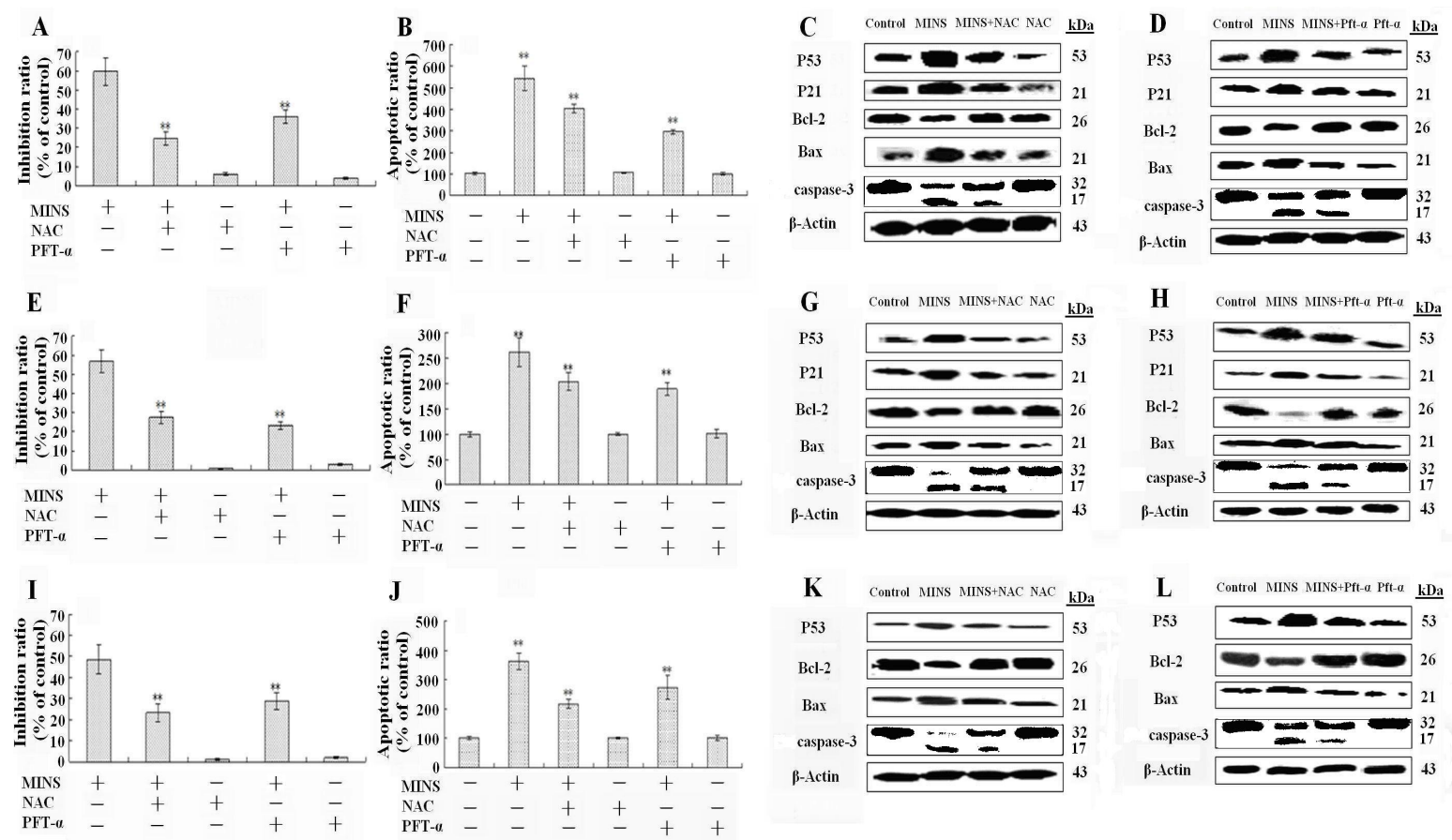

Figure 7. The effects of MINS on ROS and p53 on HT-29 cells, HCT-116 cells and Caco-2-p53 cells. HT-29 cells, HCT116 cells and Caco-2-p53 cells were separately treated with MINS $35 \mu \mathrm{M}, 30 \mu \mathrm{M}, 50 \mu \mathrm{M}$ for 36 h. The cell growth inhibitory ratio of HT-29 cells (A), HCT-116 cells (E) and Caco-2-p53 cells (I) was measured by MTT assay. The apoptotic ratio of HT-29 cells (B), HCT-116 cells (F) and Caco-2-p53 cells ( $\mathrm{J})$ was measured by Annexin V fluorometric analysis $(\mathrm{n}=$ $3,{ }^{*} \mathrm{p}<0.05,{ }^{* *} \mathrm{p}<0.01$ vs Control). Effect of NAC $(500 \mu \mathrm{M})$ on the protein expressin of p53 and other related protein in MINS- treated HT-29 cells (C), HCT-116 cells (G) and Caco-2-p53 (K) cells, the independence experiment was replicated three times. Effect of PFT- $\alpha(10 \mu \mathrm{M})$ on the protein expressin of p53 and other related protein in MINS-treated HT-29 cells (D), HCT-116 cells (H) and Caco-2-p53 (L) cells, the independence experiment was replicated three times. 
failed to induce Caco-2 (p53 null) cells apoptosis and ROS production (Supplementary Figure 2(D) and Supplementary Figure 2(E)), suggesting that ROS and p53 are necessary for MINS-induced cell apoptosis

\section{Discussion}

The occurrence and development of malignant tumor are closely related to the disorders of the intracellular polyamine homeostasis, furthermore, polyamine synthase activity and polyamine concentrations often prodigiously increased in tumor cells [5] [11]. Naphthalimide compounds, as DNA intercalating agents, had potential antitumor effects. Therefore, naphthalimide-polyamine conjugates have become one of the research focuses as antitumor drugs at present. In recent years, our laboratory is committed to the synthesis and evaluation of novel naphthalimide-polyamine conjugates as antitumor leading compounds. Some of them exert potent activity on K562 cells, MDA-MB-231 cells, HepG2 cells, BEL-7402 cells and other tumor cells than Amonafide, and also have favorable selectivity in vitro [12] [13]. NNINspm has the satisfactory identification ability about the polyamine transporter and favorable tumor targeting. Regrettably, NNINspm had obvious toxicity in therapeutic dose after evaluation in vivo [14]. So we made a further structural modification and a serial of novel naphthalimide-polyamine conjugates were synthesized. After initial screening, we found MINS exerted potent antiproliferative activity on many cancer cell lines and favourable selectivity between colorectal cancer cells and normal intestinal epithelial IEC-6 cells (data not shown). In addition, the antitumor mechanism of such compounds is infrequent in colorectal cancer. Accordingly, we elucidated the antiproliferative effects and the molecular mechanism of MINS in human colorectal cancer cell lines.

Previous data demonstrated that $5 \mathrm{~d}$, a naphthalimide-polyamine conjugate, induced murine melanoma B16 cell apoptosis via mitochondrial pathway and death receptor pathway [13]. NPC-16, another naphthalimide-polyamine conjugate, could induce HepG2 cells apoptosis and autophagy [8]. However, MINS only induced HCT-116 cells, HT-29 cells and Caco-2-p53 cells apoptosis but not autophagy (data not shown). Further, MINS induced apoptosis via mitochondrial pathway and death receptor pathway in HT-29 cells and Caco-2-p53 cells, but only mitochondrial pathway in HCT-116 cells and the apoptosis is caspase dependent. These findings suggested that naphthalimide-polyamine conjugates mediated apoptotic mechanism depend on cell types and the structural modification could change the cell death mode. Bcl-2 family members usually regulate mitochondrial apoptosis pathway [15]. In three colorectal cancer cells, MINS induced Bcl-2 down-regulation and Bax up-regulation, suggesting that Bcl-2 family members were involved in MINS-mediated cell apoptosis. Consequently, MINS induced MMP decrease and cytochrome $c$ release from mitochondria to cytoplasm.

The generation of ROS in tumor cells, especially in the key parts of the cells, such as the cell membrane, mitochondrial membrane and the nucleus, can change the cellular redox state of equilibrium, start intracellular signal transduction, affect the proliferation of cells, and also regulate many apoptotic relative molecules, such as Bax, Caspase-3 and Caspase-8 [16]. This apoptotic effect is similar with 7c, an Amonafide analogue, which induced human Hela cervix carcinoma cell apoptosis via enhancement ROS generation [17]. Our results demonstrated that NAC not only reversed the apoptotic effects of MINS but also some apoptotic relative proteins. In addition, we also elucidated that the source of MINS-mediated ROS was predominantly due to the mitochondria electron transport chain. In addition, we previously also found that mononaphthalimide-spermidine conjugate could induce ROS accumulation and then lead to Hela cell apoptosis [18]. Therefore, ROS generation may be the common mechanism in naphthalimide derivates-mediated tumor cell apoptosis.

The p53 is involved in the apoptosis progress regulation through the causation of the cell cycle arrest and the regulation of Bcl-2 family [19]. The p21 is a Cyclin-dependent kinase inhibitor encoded by p53 downstream genes [20]. Our results demonstrated that MINS induced the up-regulation of p53 in three colorectal cancer cells in a time- and dose-dependent manner. Furthermore, pretreatment with pifithrin- $\alpha$ could attenuate MINS-mediated p53 up-regulation, and also regulate their downstream proteins, such as p21, Bcl-2 and Bax, suggesting that MINS-mediated apoptosis was dependent on p53 signal pathway. In addition, after treatment with effective concentrations which exerted antiproliferative activity, MINS only induced Caco-2 (p53 null) cells necrosis, but not apoptosis. Furthermore, MINS failed to induce significantly ROS production, suggesting that MINS-mediated apoptosis was dependent on p53.

\section{Conclusion}

In summary, MINS-induced cell apoptosis was associated with p53, ROS and Bcl-2 family members were also 
involved in this apoptotic process in HCT-116, HT-29, Caco-2-p53 human colon cancer cells. These results suggested that MINS merited further investigation and showed the therapeutic value in the treatment of cancers depending on p53 signaling pathway.

\section{Acknowledgements}

This work was supported by NSFC-Henan talented man train union fund (No. U1204829) and Program for Science and Technology Innovation Talents in Universities of Henan Province (No. 14HASTIT033).

\section{Conflicts of Interest}

The authors declared that they have no competing interest.

\section{References}

[1] Cantero-Muñoz, P., Urién, M.A. and Ruano-Ravina, A. (2011) Efficacy and Safety of Intraoperative Radiotherapy in Colorectal Cancer: A Systematic Review. Cancer Letters, 306, 121-133. http://dx.doi.org/10.1016/j.canlet.2011.02.020

[2] Nelson, S.M., Ferguson, L.R. and Denny, W.A. (2004) DNA and the Chromosome-Varied Targets for Chemotherapy. Cell Chromosome, 3, 2. http://dx.doi.org/10.1186/1475-9268-3-2

[3] Braña, M.F. and Ramos, A. (2001) Naphthalimides as Anticancer Agents: Synthesis and Biological Activity. Current Medicinal Chemistry-Anti-Cancer Agents, 1, 237-255. http://dx.doi.org/10.2174/1568011013354624

[4] Nasizadeh, S., Myhre, L., Thiman, L., Alm, K., Oredsson, S. and Persson, L. (2005) Importance of Polyamines in Cell Cycle Kinetics as Studied in a Transgenic System. Experimental Cell Research, 308, 254-264. http://dx.doi.org/10.1016/j.yexcr.2005.04.027

[5] Basuroy, U.K. and Gerner, E.W. (2006) Emerging Concepts in Targeting the Polyamine Metabolic Pathway in Epithelial Cancer Chemoprevention and Chemotherapy. Journal of Biochemistry, 139, 27-33. http://dx.doi.org/10.1093/jb/mvj022

[6] Tian, Z.Y., Ma, H.X., Xie, S.Q., Wang, X. and Zhao, J. (2008) Synthesis, DNA Binding and Topoisomerase Inhibition of Mononaphthalimide Homospermidine Derivatives. Chinese Chemical Letters, 19, 509-512. http://dx.doi.org/10.1016/j.cclet.2008.03.006

[7] Xie, S., Wang, J., Zhang, Y. and Wang, C. (2010) Antitumor Conjugates with Polyamine Vectors and Their Molecular Mechanisms. Expert Opinion on Drug Delivery, 7, 1049-1061. http://dx.doi.org/10.1517/17425247.2010.504205

[8] Xie, S.Q., Li. Q., Zhang, Y.H., Wang, J.H., Mei, Z.H., Zhao, J. and Wang, C. (2011) NPC-16, a Novel Naphthalimide-Polyamine Conjugate, Induced Apoptosis and Autophagy in Human Hepatoma HepG2 Cells and Bel-7402 Cells. Apoptosis, 16, 27-34. http://dx.doi.org/10.1007/s10495-010-0537-1

[9] Wang, Y., Zhang, X., Zhao, J., Xie, S. and Wang, C. (2012) Nonhematotoxic Naphthalene Diimide Modified by Polyamine: Synthesis and Biological Evaluation. Journal of Medicinal Chemistry, 55, 3502-3512. http://dx.doi.org/10.1021/jm300168w

[10] Xie, S.Q., Wu, Y.L., Cheng, P.F., Wang, M.W., Liu, G.C., Ma, Y.F., Zhao, J. and Wang, C.J. (2007) A Novel Homospermidine Conjugate Inhibits Growth and Induces Apoptosis in Human Hepatoma Cells. Acta Pharmacologica Sinica, 28, 1827-1834. http://dx.doi.org/10.1111/j.1745-7254.2007.00639.x

[11] Braña, M.F., Cacho, M., García, M.A., de Pascual-Teresa, B., Ramos, A., Acero, N., Llinares, F., Muñoz-Mingarro, D., Abradelo, C., Rey-Stolle, M.F. and Yuste, M. (2002) Synthesis, Antitumor Activity, Molecular Modeling and DNA Binding Properties of a New Series of Imidazonaphthalimides. Journal of Medicinal Chemistry, 45, 5813-5816. http://dx.doi.org/10.1021/jm020950q

[12] Tian, Z.Y., Xie, S.Q., Mei, Z.H., Zhao, J., Gao, W.Y. and Wang, C.J. (2009) Conjugation of Substituted Naphthalimides to Polyamines as Cytotoxic Agents Targeting the Akt/mTOR Signal Pathway. Organic Biomolecular Chemistry, 7, 4651-4660. http://dx.doi.org/10.1039/b912685f

[13] Tian, Z.Y., Xie, S.Q., Du, Y.W., Ma, Y.F., Zhao, J., Gao, W.Y. and Wang, C.J. (2009) Synthesis, Cytotoxicity and Apoptosis of Naphthalimide Polyamine Conjugates as Antitumor Agents. European Journal of Medicinal Chemistry, 44, 393-399. http://dx.doi.org/10.1016/j.ejmech.2008.02.044

[14] Xie, S.Q., Li, Q., Zhang, Y.H., Wang, J.H., Zhao, J. and Wang, C.J. (2010) NNINspm, Naphthalimide-Polyamine Conjugate, Induces Hepatoma HepG2 Cell Apoptosis via PI3K/Akt Pathways. Chinese Pharmacological Bulletin, 26,169-174.

[15] Wasilewski, M. and Scorrano, L. (2009) The Changing Shape of Mitochondrial Apoptosis. Trends in Endocrinology Metabolism, 20, 287-294. http://dx.doi.org/10.1016/j.tem.2009.03.007 
[16] Circu, M.L. and Aw, T.Y. (2010) Reactive Oxygen Species, Cellular Redox Systems and Apoptosis. Free Radical Biology Medicine, 48, 749-762. http://dx.doi.org/10.1016/j.freeradbiomed.2009.12.022

[17] Shen, K., Sun, L., Zhang, H., Xu, Y., Qian, X., Lu, Y., Li, Q., Ni, L. and Liu, J. (2013) A ROS-Mediated Lysosomal-Mitochondrial Pathway Is Induced by a Novel Amonafide Analogue, 7c, in Human Hela Cervix Carcinoma Cells. Cancer Letters, 333, 229-238. http://dx.doi.org/10.1016/j.canlet.2013.01.038

[18] Yang, L., Zhao, J., Zhu, Y., Tian, Z. and Wang, C. (2011) Reactive Oxygen Species (ROS) Accumulation Induced by Mononaphthalimide-Spermidine Leads to Intrinsic and AIF-Mediated Apoptosis in Hela Cells. Oncology Reports, 25, 1099-1107. http://dx.doi.org/10.3892/or.2011.1173

[19] Gonmes, N.P. and Espinosa, J.M. (2010) Gene-Specific Repression of the p53 Target Gene PUMA via Intragenic CTCF-Cohesin Binding. Genes Development, 24, 1022-1034. http://dx.doi.org/10.1101/gad.1881010

[20] Al-Hai, L., Blackshear, P.J. and Khabar, K.S. (2012) Regulation of p21/CIP1/WAF-1 Mediated Cell-Cycle Arrest by RNase L and Tristetraprolin and Involvement of AU-Rich Elements. Nucleic Acids Research, 40, 7739-7752. http://dx.doi.org/10.1093/nar/gks545

\section{Supplementary}

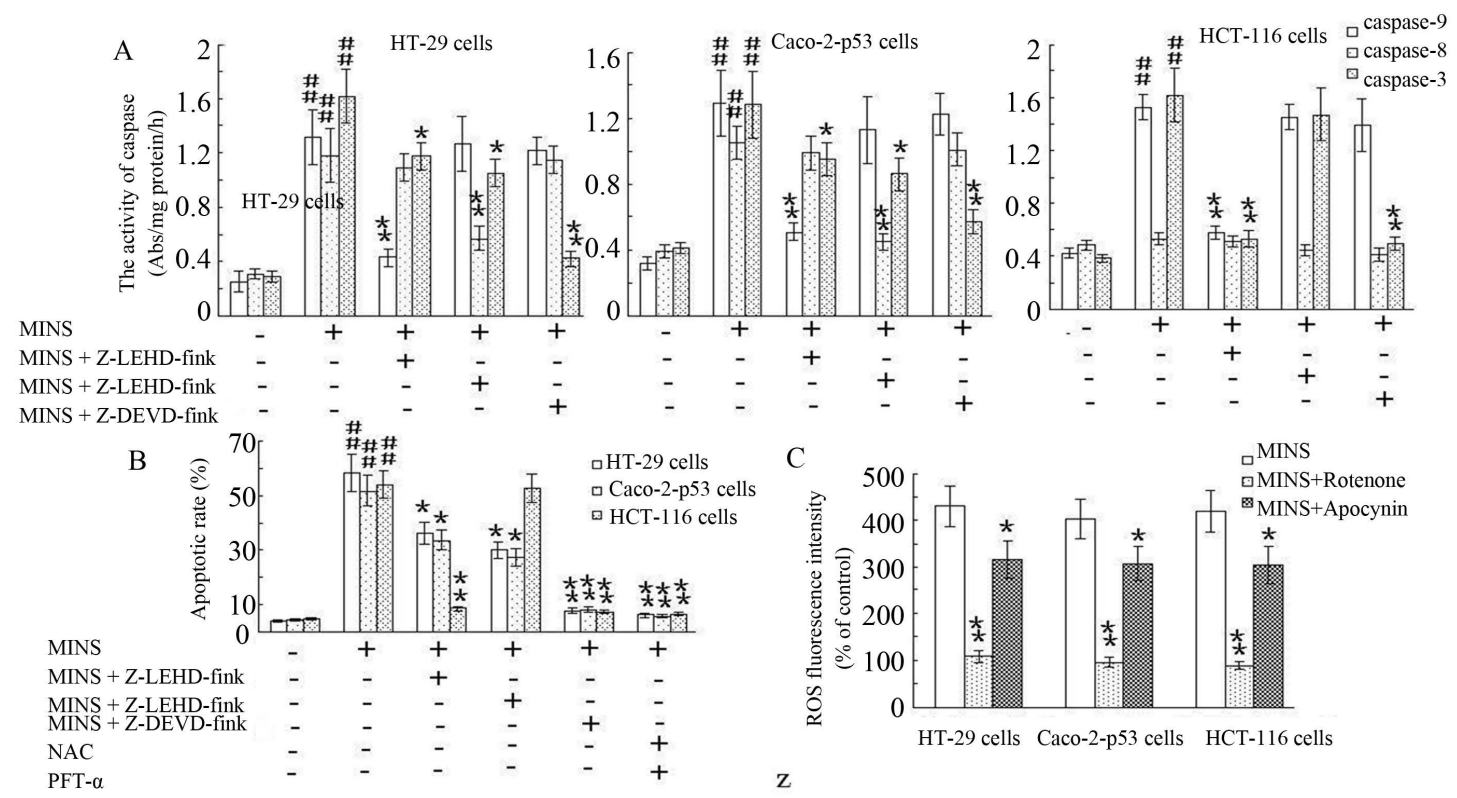

Supplementary Figure 1. The effects of MINS on Caspase activation, cell apoptosis and ROS generation in HT-29 cells, HCT-116 cells and Caco-2-p53 cells. HT-29 cells, HCT-116 cells and Caco-2-p53 cells were separately treated with MINS $35 \mu \mathrm{M}, 30 \mu \mathrm{M}, 50 \mu \mathrm{M}$ for $12 \mathrm{~h}$. (A) The activity of caspase-3, caspase-8, caspase-9 after treatment with MINS $12 \mathrm{~h}$ present or absent Z-DEVD-FMK $(20 \mu \mathrm{M})$, Z-IETD-FMK $(20 \mu \mathrm{M})$ and Z-LEHD-FMK $(20 \mu \mathrm{M})$, respectively. Means \pm SD, $\mathrm{n}=3$, Note: ${ }^{*} \mathrm{p}<0.05,{ }^{* *} \mathrm{p}<0.01$ vs MINS group; ${ }^{\# \#} \mathrm{p}<0.01$ vs Control. (B) The apoptotic rate of HT-29 cells, HCT-116 cells and Caco-2-p53 cells after treatment with MINS $12 \mathrm{~h}$ present or absent Z-DEVD-FMK $(20 \mu \mathrm{M})$, Z-IETD-FMK $(20 \mu \mathrm{M})$ and Z-LEHD-FMK $(20 \mu \mathrm{M})$, NAC $(500 \mu \mathrm{M})$ and PTF- $\alpha(10 \mu \mathrm{M})$, respectively. Means $\pm \mathrm{SD}, \mathrm{n}=3$, Note: ${ }^{*} \mathrm{p}<0.05,{ }^{* *} \mathrm{p}<0.01$ vs MINS group; ${ }^{\# \#} \mathrm{p}<0.01$ vs Control. (C) The ROS fluorescence intensity of HT-29 cells, HCT-116 cells and Caco-2-p53 cells after treatment with MINS $12 \mathrm{~h}$ present or absent Rotenone $(25 \mathrm{nM})$ and Apocynin $(100 \mu \mathrm{M})$, respectively. Means \pm $\mathrm{SD}, \mathrm{n}=3$, Note: ${ }^{*} \mathrm{p}<0.05,{ }^{* *} \mathrm{p}<0.01$ vs MINS group. 

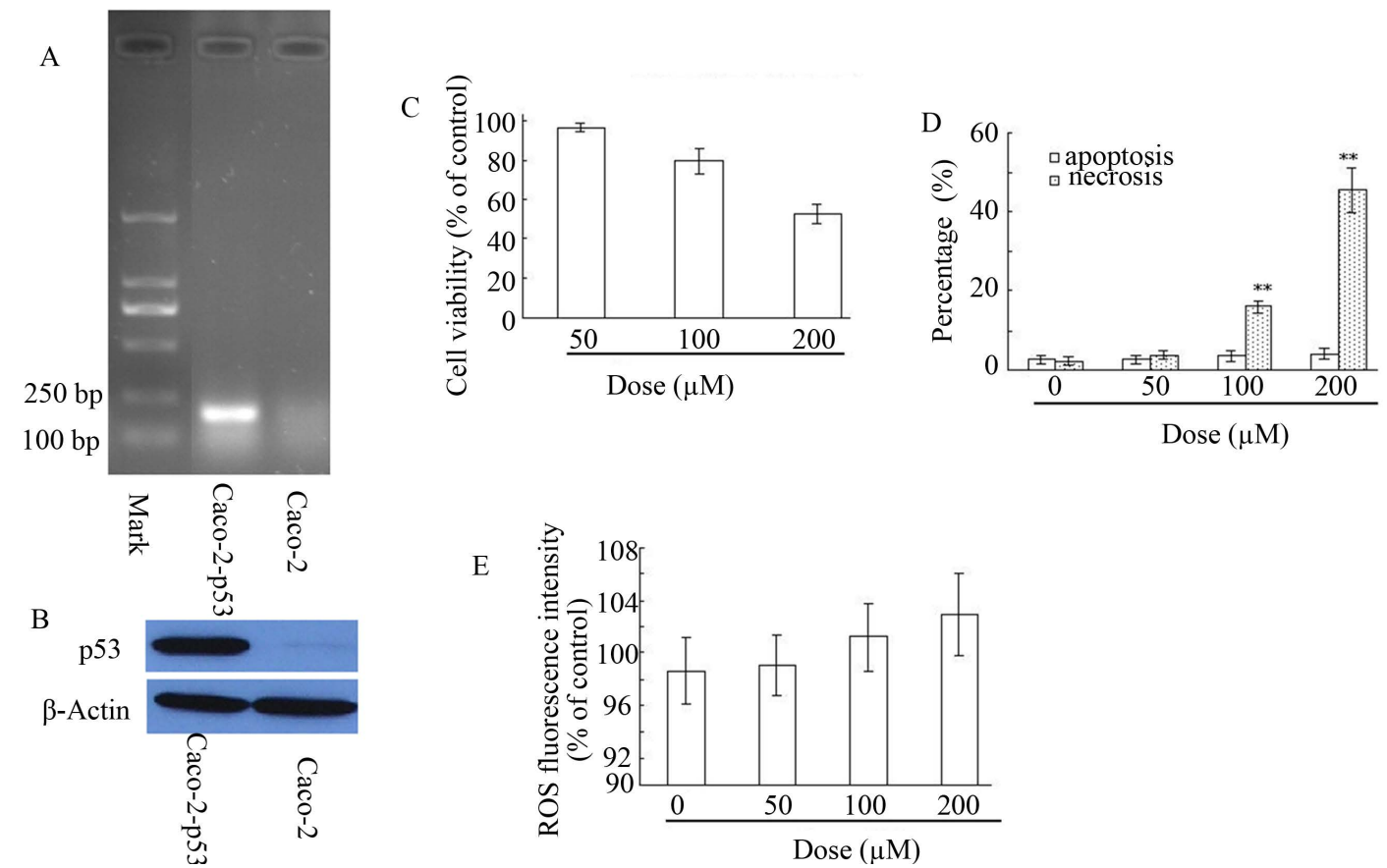

Supplementary Figure 2. Wtp53 gene was transfected into Caco-2 cells and the effects of MINS on Caco-2-p53 cells apoptosis and ROS generation were separately treated with MINS $(50 \mu \mathrm{M}, 100 \mu \mathrm{M}, 200 \mu \mathrm{M})$ for 24 h. The expression of p53 mRNA (A) and protein (B) were detected in Caco-2-p53 cells and Caco-2 cells. (C) The antiproliferative activity of MINS was evaluated using MTT assay on Caco-2-p53 cells after treatment with $50 \mu \mathrm{M}, 100 \mu \mathrm{M}$ and $200 \mu \mathrm{M}$ for $24 \mathrm{~h}$ (means $\pm \mathrm{SD}, \mathrm{n}=3$ ). (D) MINS-mediated apoptosis and necrosis were evaluated on Caco-2-p53 cells after treatment with 50 $\mu \mathrm{M}, 100 \mu \mathrm{M}$ and $200 \mu \mathrm{M}$ for $24 \mathrm{~h}$, means $\pm \mathrm{SD}, \mathrm{n}=3,{ }^{* *} \mathrm{p}<0.01$ vs Control. (E) MINS-mediated ROS production was evaluated on Caco-2-p53 cells after treatment with $50 \mu \mathrm{M}, 100 \mu \mathrm{M}$ and $200 \mu \mathrm{M}$ for $24 \mathrm{~h}$, means $\pm \mathrm{SD}, \mathrm{n}=3$. 
Scientific Research Publishing (SCIRP) is one of the largest Open Access journal publishers. It is currently publishing more than 200 open access, online, peer-reviewed journals covering a wide range of academic disciplines. SCIRP serves the worldwide academic communities and contributes to the progress and application of science with its publication.

Other selected journals from SCIRP are listed as below. Submit your manuscript to us via either submit@scirp.org or Online Submission Portal.
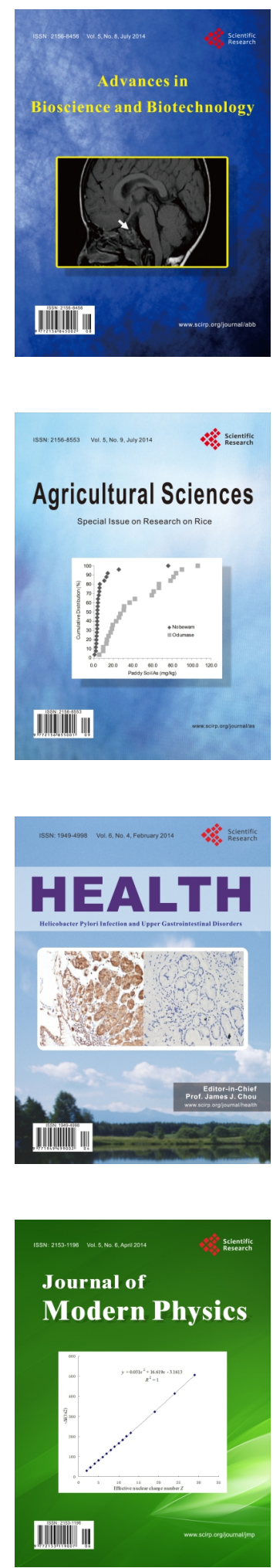
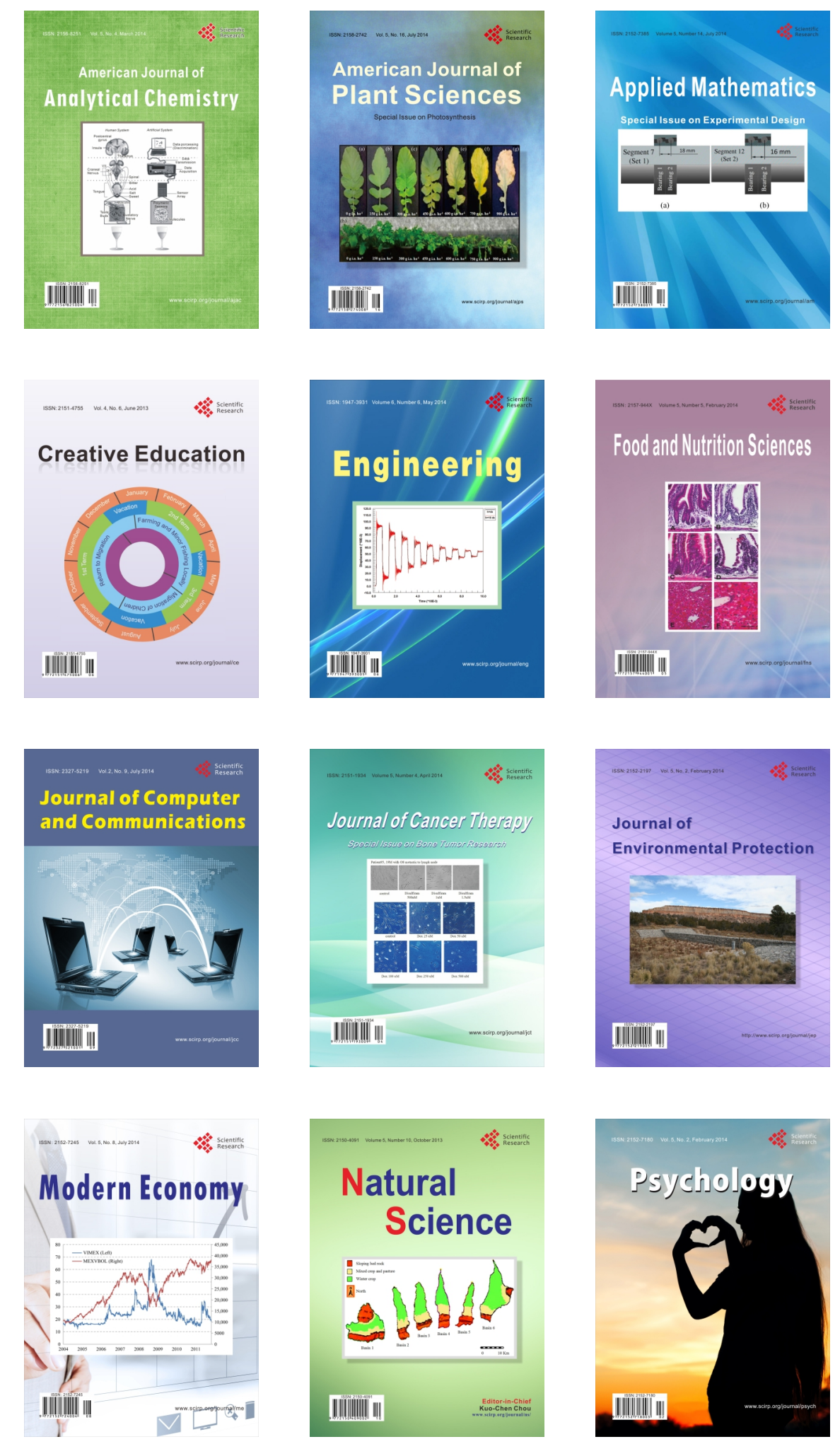